\title{
Analytical design of the human bronchial tree for healthy patients and patients with obstructive pulmonary diseases
}

\author{
Medvedev A.E. ${ }^{1,2 *}$, Gafurova P.S. , $^{* * *}$ \\ ${ }^{1}$ Khristianovich Institute of Theoretical and Applied Mechanics SB RAS, Novosibirsk, Russia \\ ${ }^{2}$ Novosibirsk State University, Novosibirsk, Russia
}

\begin{abstract}
The study is aimed at the analytical design of the full human bronchial tree for healthy patients and patients with obstructive pulmonary diseases. Analytical formulas for design of the full bronchial tree are derived. All surfaces of the bronchial tree are matched with the second-order smoothness (there are no acute angles or ribs). The geometric characteristics of the human bronchial tree in the pathological case are modeled by a "starry" shape of the inner structure of the bronchus; the pathology degree is defined by two parameters: bronchus constriction level and degree of distortion of the cylindrical shape of the bronchus. Closed analytical formulas allow the human bronchial tree of an arbitrary complexity (up to alveoli) to be designed; moreover, the parametric dependences make it possible to specify any desirable degree of airway obstruction.
\end{abstract}

Key words: bronchial tree, simulation, human lungs, respiratory system, bifurcation.

\section{INTRODUCTION}

The human respiratory system consists of the upper (nose, nasal pharynx, and larynx) and lower (trachea, bronchial tree, and alveoli) air passages. The human bronchial tree has a complicated tree-like structure. There are 24 branching points (bifurcations) from the trachea to alveoli in the bronchial tree. Generally speaking, the cross-sectional shapes of individual bronchi differ from circumferences. In pathological situations (e.g., asthma), the bronchi are constricted, and their cross-sectional shapes change.

One of the main difficulties arising in lung simulations is an appropriate description of the lung geometry. For simulating the air flow in the human bronchial tree, it is necessary to design its three-dimensional model with bifurcations. The main difficulty in design of the bronchial tree bifurcation is a correct description of the carinal rounding of the diverging bronchi. Nonalgorithmic construction of the carinal rounding (e.g., simple smoothing of sharp ribs) requires many manual operations and careful design of a branching bronchial tree. The quality of the designed bronchial tree affects the computation accuracy and speed. Because of the presence of ribs and non-smooth matching of bifurcations and bronchi in the model, the air flow in computations becomes turbulent. As a result, the drag force generated by the air flow passing through the bronchial tree increases, which affects the results.

The second difficulty in computer simulations of lungs is the geometric growth of the number of bronchi: the bronchial tree begins from one trachea and ends approximately with $2^{23}=8388608$ alveoli, while the bronchus scale decreases approximately by a factor of 240 . The third difficulty in modeling human lungs is the constriction of the cross section of pathological bronchi. The cross section of healthy bronchi is close to a circle, whereas the cross-

*medvedev@itam.nsc.ru

***polina_g96@mail.ru 
sectional area of pathological bronchi decreases, and the bronchus cross section acquires a "starry" shape.

In most studies, the bronchial tree design is based on the model [1], which describes the basic geometric parameters of the human bronchial tree.

The flow in a large bifurcation was studied in [2, 3], where a bifurcation model was developed (Fig. 1). Detailed formulas for geometric construction of bifurcations were derived in [2]. The overall view of this bifurcation is appreciably different from the anatomic structure of the bronchial tree bifurcations. The output bronchi enter the bifurcation system at an acute angle $[2,3]$, and there are sharp ribs, which contradicts the smooth contours of real bronchi.
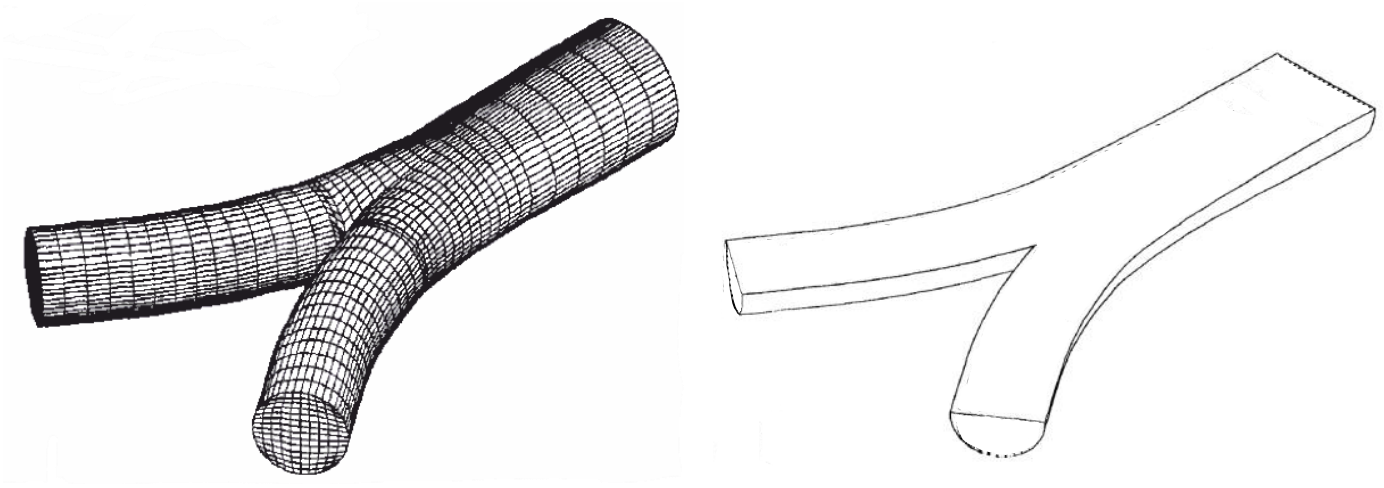

Fig. 1. Bifurcation of the bronchial tree from $[2,3]$.

A detailed mathematical description of the bifurcation geometry was given in [4] (Fig. 2), where eight pages were devoted to the description of the algorithm used to construct an individual bifurcation. An advantage of that paper is the design of an asymmetric bifurcation. An individual branch of the bronchial tree up to 11 generations was constructed. This model is based on physiologically realistic geometry of the bronchial tree bifurcation [5]. The algorithmic method of constructing a smooth bifurcation and bronchial tree as a whole, which was proposed in [4] and further developed in [5], was fitted to a particular computer code for constructing three-dimensional objects (GAMBIT preprocessor for the FLUENT software system and UNIGRAHICS computer simulation code).
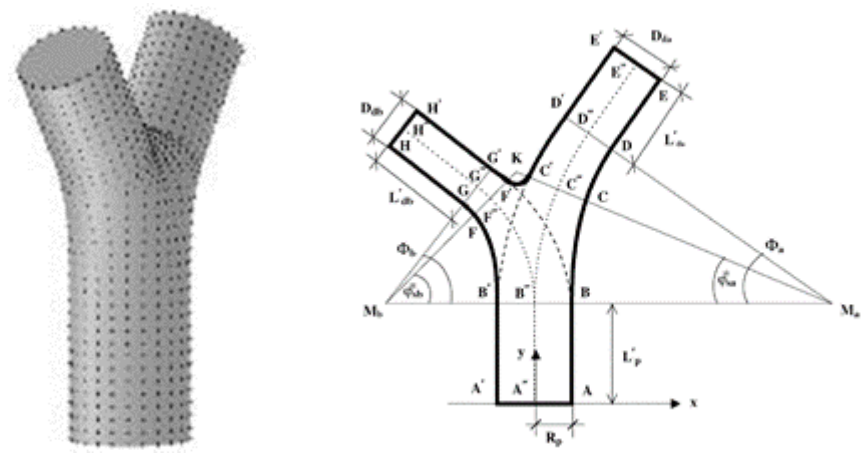

Fig. 2. Bronchial tree bifurcation from [4].

Based on statistical processing of tomography data, a model of the human bronchial tree was developed in [6] (Fig. 3). The resultant graph of the bronchial tree contains up to seven generations. 

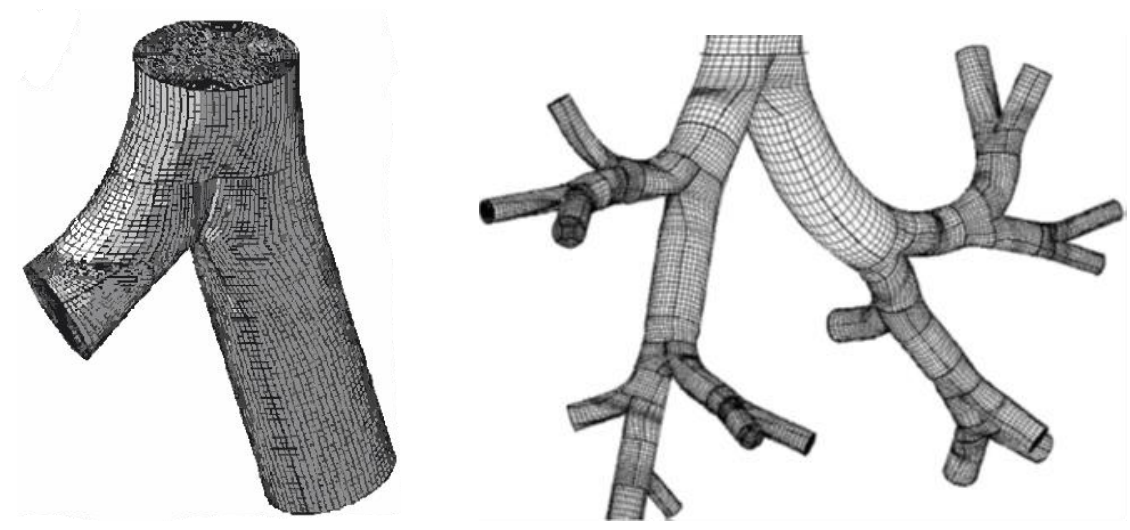

Fig. 3. Bronchial tree bifurcation from [6].

Two torus configurations were used to design an individual bifurcation in [7-9] (Fig. 4). Geometric construction of the upper and lower torus configurations was shown in [8], but no formulas were provided. The issue of the surface covering the gap between the torus configurations remained open (most probably, a smoothing surface in a 3D simulation code was constructed). The bifurcation design in [8] was based on manual algorithmic construction of the bifurcation in a some code for 3D simulations. One branch of the bronchial tree up to the 16-th generation was designed.
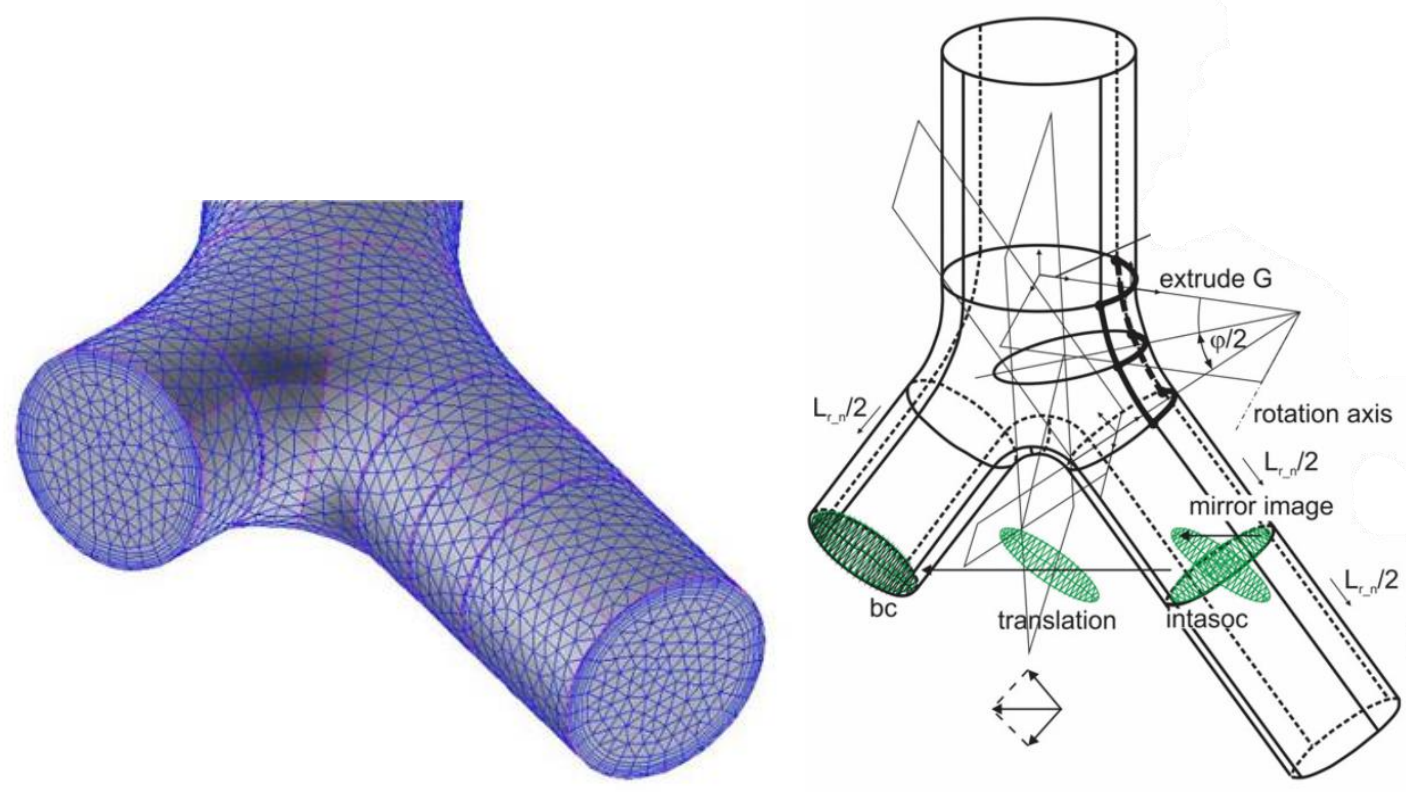

Fig. 4. Design of the bronchial tree bifurcation from $[7,8]$.

Based on the anatomic diagram [6], an asymmetric bronchial tree up to the 17-th generation was constructed in $[10,11]$. The air flow in this tree was numerically studied. Simulations were performed with the use of the ANSYS Fluent software system (https://www.ansys.com/). The computational model of the bronchial tree contained 1453 bronchi, and the grid included $6.744 \cdot 10^{6}$ unstructured four-sided elements. Air was considered as a viscous incompressible fluid. The air flow rate was 28.3 liters per minute, and the computations were performed within the framework of the turbulent flow model. A typical time of one computation on computers of the supercomputer center was 50 hours.

Some part of the asymmetric human bronchial tree was constructed in $[12,13]$ (the left and right lungs consist of three and two lobes, respectively). The bronchial tree geometry was based 
on the model [1] corrected on the basis of information derived from medical reference books and consultations with specialists (practicing physicians). The three-dimensiona geometry was designed by using a universal CAD-editor AnsysDesignModeler (Fig. 5). There were no other details of bifurcation construction in $[12,13]$.

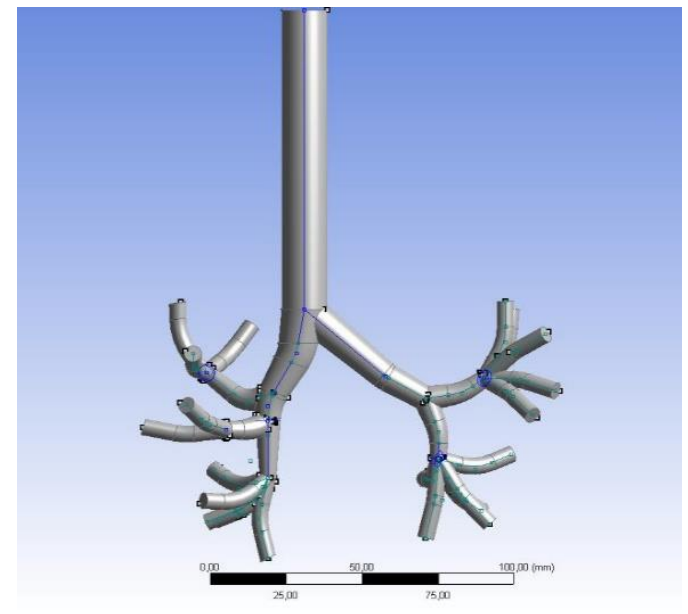

Fig. 5. Three-dimensional geometry of an asymmetric bronchial tree from [12].

In [14], the three-dimensional structure of large airways up to the 16-th generation was reconstructed on the basis of tomography data on human lungs. A one-dimensional model was used for simulating smaller airways. The proposed model was applied to study the flow parameters of the inhaled and expired air under normal breathing conditions.

In the above-cited publications, the main aspect of design of the 3D human bronchial tree was the algorithmic aspect of the process of bifurcation modeling in 3D simulation codes. Construction of an individual bifurcation requires significant time expenses and manual operations. The algorithm of matching of individual bifurcations was not described and requires manual fitting in 3D simulation codes. In all paper cited above, only bronchi with a circular cross section were considered. When a constriction of the circular cross section of a bronchus has to be described, the algorithmic design of the bronchi with new parameters has to be repeated. The full (up to the 23 generations) branch of the bronchial tree was constructed in none of the above-cited papers because of the large labor expenses of constructing an individual bifurcation.

The goal of the present study is to develop an analytical model of the human lung, which ensures effective simulation of the air flow in the healthy and pathological bronchial trees from the input bronchus to alveoli. The analytical model of the bifurcation contains closed formulas for constructing the full human bronchial tree from the 0-th to 23-rd bifurcation inclusive. Analytical formulas allow easy modeling of lung system pathologies caused by the "starry" constriction of the bronchi. Moreover, analytical formulas allow one to avoid tedious manual operations of constructing each individual bifurcation in 3D simulation codes, their matching and smoothing (the procedure of manual design of the pathological bifurcation is especially complicated). The proposed analytical formulas of bronchial tree construction allow numerical simulations to be performed for bronchial trees with an arbitrary number of generation (provided that appropriate computational resources are available) for normal and pathological cases.

\section{HISTOLOGICAL STRUCTURE OF BRONCHI}

The cross-sectional shape of the bronchi differs from a circumference even if there are no pathological effects. Figure 6 shows the microphotographs of the bronchus cross section. The 
inner boundary has a starry shape owing to the presence of epithelium cells. The epithelium covers the muscular fibers located underneath. The outer shape of the bronchi is supported by cartilaginous plates, which are closed for large bronchi and non-closed for small bronchi. Contraction of muscular fibers leads to constriction of the inner cross section of the bronchi, which is observed, in particular, in the case of an asthmatic attack (Fig. 7).

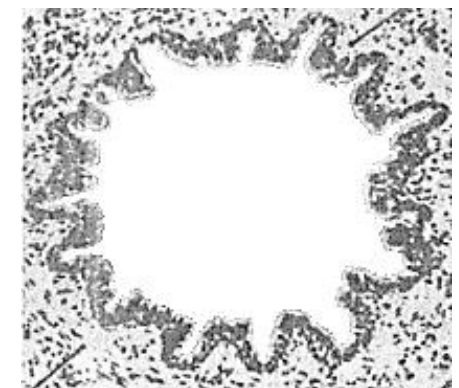

a)

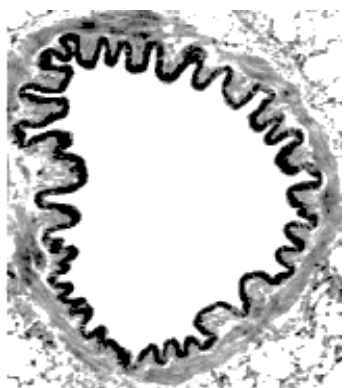

b)

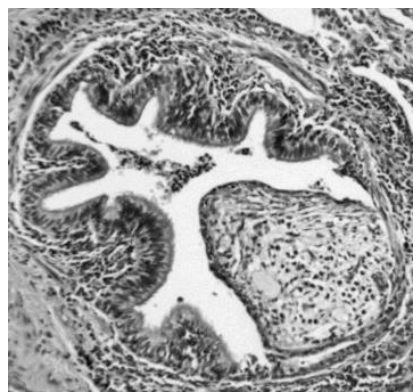

c)

Fig. 6. Microphorographs of the inner structure of the bronchi: (a) large bronchiole in a child's lung [15]; (b) human bronchiole [16]; (c) bronchiole in the case of a chronic obstructive pulmonary disease [17].

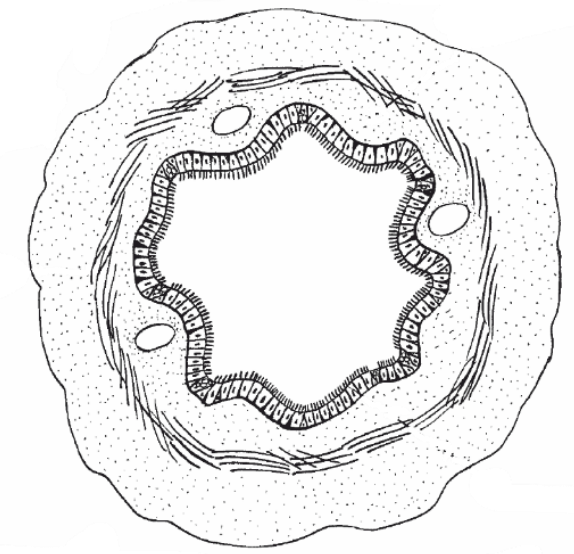

a)

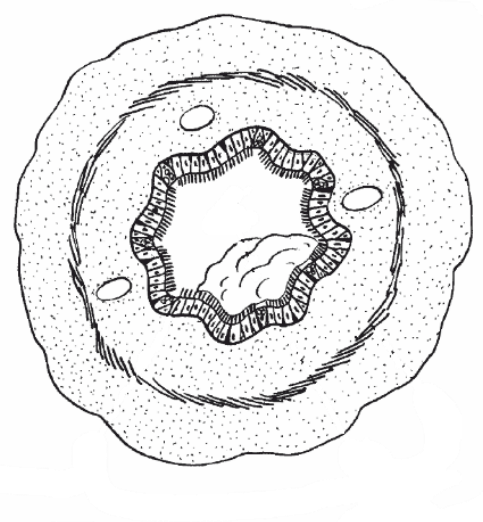

b)

Fig. 7. Cross section of the normal bronchus (a) and of the bronchus of a patient with asthma (b). Schematic pattern from [18].

In constructing the bronchial tree model, one has to take into account the real (or close to real) shape of the inner structure of the bronchi because the inner shape of the bronchus significantly affects the air flow characteristics in the bronchi.

\section{BRONCHIAL TREE PARAMETERS}

Design of the mathematical model of the human bronchial tree is a complicated and laborconsuming task. The human bronchial tree has a complicated structure consisting of bronchi and connecting bifurcations (structures where the input bronchus branches into two output bronchi). The three-dimensional geometry of the bronchial tree is generated on the basis of the morphometric data on human lungs [1]. Generally speaking, the bronchial tree is asymmetric, and the airway sizes of different people can be significantly different. A brief review of the human bronchial tree characteristics from various studies can be found in [12].

As the initial morphometric data for constructing the analytical model of the bronchial tree, we use a simple model of a symmetric bronchial tree [1]. It will be demonstrated that the resultant bronchial tree can be easily extended to other morphometric data of human lungs. According to [1], the human bronchial tree has 24 generations of bifurcations. The bifurcation number $n$ (or the number of the bronchial tree generation) varies from 0 to 23 . The parameters 
of symmetric dichotomy (symmetric bronchial tree model [1]) are defined by the following formulas [9]:

- input bronchus radius (in $\mathrm{mm}$ )

$$
R_{n}= \begin{cases}9 e^{-0.388 n}, & n \leq 3 \\ 6.5 e^{-(0.2929-0.00624 n) n}, & n>3\end{cases}
$$

- input bronchus length (in $\mathrm{mm}$ )

$$
L_{n}= \begin{cases}120 e^{-0.92 n}, & n \leq 3 \\ 25 e^{-0.17 n}, & n>3\end{cases}
$$

Functions (1) and (2) are plotted in Figure 8.

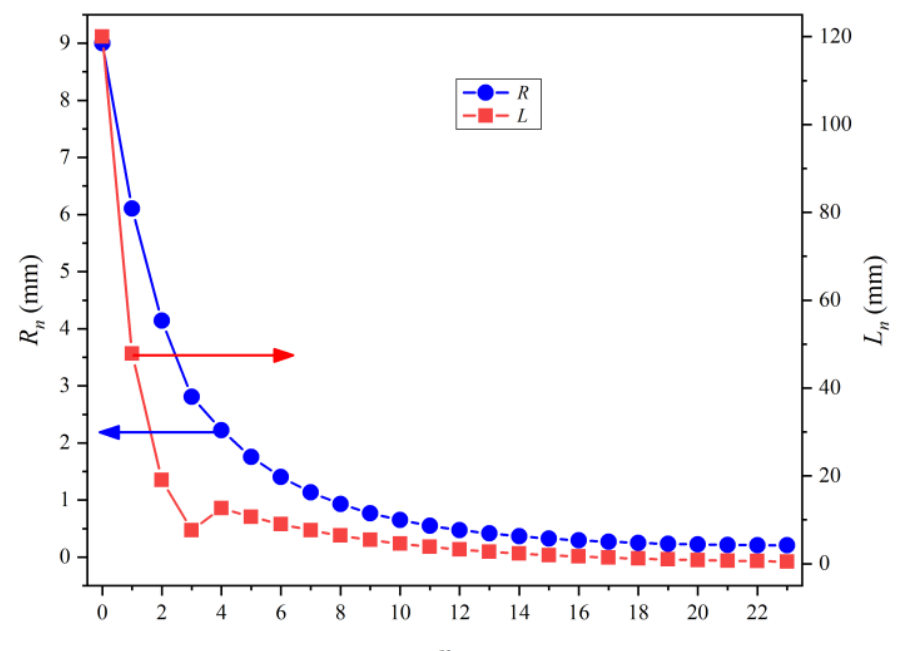

Fig. 8. Radius $R_{n}$ and length $L_{n}$ of the bronchi versus the bifurcation number $n$ for the symmetric bronchial tree model (1), (2).

The air density is $\rho=1.23 \mathrm{~kg} / \mathrm{m}^{3}$, and the dynamic viscosity coefficient is $\mu=1.79 \mathrm{~Pa} \cdot \mathrm{s}$. The air flow rate for an adult at rest is $Q=51 / \mathrm{min}$ and increases to $140 \mathrm{l} / \mathrm{min}$ in a middledistance race. The maximum decrease in pressure in the human lungs is $\Delta p \approx 35 \mathrm{~mm} \mathrm{H}_{2} \mathrm{O} \approx 343 \mathrm{~Pa}[12,13]$. Thus, the maximum difference in pressure in the human lungs is $0.338 \%$ of atmospheric pressure.

The Reynolds number of the air flow in the bronchus of the $n$-th generation is calculated by the formula

$$
\operatorname{Re}_{n}=\frac{2 \rho U_{n} R_{n}}{\mu}=\frac{\rho Q}{2^{n-1} \pi \mu R_{n}}
$$

where $U_{n}=\frac{Q}{2^{n} \pi R_{n}^{2}}$ is the mean velocity at the output of the $n$-th bronchus. The calculated values of the Reynolds number are shown in Figure 9. The critical Reynolds number for a circular tube with smooth walls is 2300 . Figure 9 shows that the Reynolds numbers are higher than the critical value only in some large bronchi with large flow rates. Thus, the flow in the bronchial tree is laminar, except for several large bronchi with large flow rates. The flow in the human bronchial tree for a person at rest is laminar in terms of the criterion of the critical Reynolds number. 
The turbulent and laminar flows generate different levels of resistance in the tube flow, which are described by the following formulas (the Blasius formula is given for the turbulent flow):

$$
\lambda_{\text {lam }}=64 / \operatorname{Re}, \lambda_{\text {turb }}=0.3164 / \operatorname{Re}^{0.25} .
$$

The pressure difference in the tube with the turbulent flow is greater than that in the laminar flow (with an identical flow rate):

$$
\Delta p_{\text {turb }} / \Delta p_{\text {lam }}=\lambda_{\text {turb }} / \lambda_{\text {lam }}
$$

For the critical Reynolds number, the ratio of the pressure differences is equal to 1.64. Thus, a greater pressure difference is required for the turbulent flow to ensure an identical air flow rate in the human lungs with the laminar flow. Most probably, for the long years of evolution, the human organism generated the bronchial tree structure that ensures a laminar flow of air in the bronchi. Therefore, in our opinion, the air flow in the human bronchial tree has a laminar character.

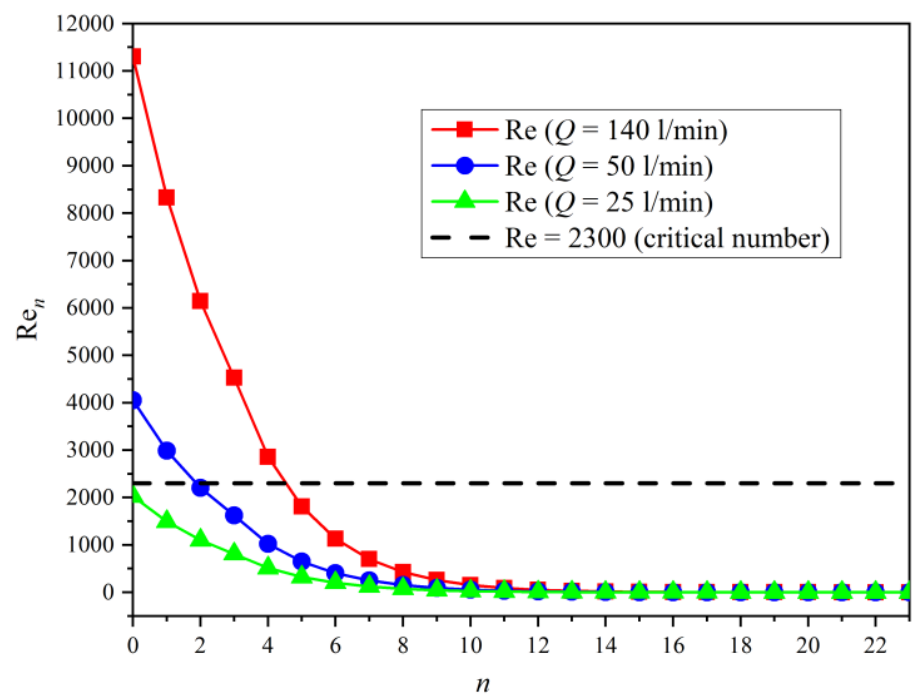

Fig. 9. Reynolds number $\operatorname{Re}_{n}$ at the output of the $n$-th bifurcation for three values of the air flow rate. $\operatorname{Re}=2300$ is the critical Reynolds number for a circular tube.

\section{MODELING OF THE HEALTHY AND PATHOLOGICAL BRONCHUS SHAPES}

The function that describes the cross-sectional shape of the bronchus shape of a healthy person and a patient with obstructive pulmonary diseases is written as

$$
f(\varphi)=\left[C-s_{\mathrm{ob}} \sin ^{2}\left(\frac{n_{\mathrm{ed}}}{2} \varphi\right)\right] \sqrt{A_{\mathrm{bronch}}},
$$

where $A_{\text {bronch }}$ is the degree of reduction of the cross-sectional area of the obstructive bronchus as compared to the healthy bronchus $\left(0<A_{\text {bronch }} \leq 1\right), s_{\mathrm{ob}}$ is the normalized height of the rays of the starry inner shape of the bronchus $\left(0<s_{\mathrm{ob}} \leq 1\right)$, and $n_{\mathrm{ed}}=4 i_{\mathrm{ed}}$ is the number of rays of the starry inner shape of the bronchus $\left(i_{\mathrm{ed}} \geq 0\right)$. The number of rays should be multiple of four for the neighboring bronchi to be matched with each other after turning the structure by $\pi / 2$ (as will be demonstrated below). The parameter $C$ is chosen from the condition that the crosssectional area of the bronchus remains unchanged regardless of the changes in the parameters $s_{\mathrm{ob}}$ and $n_{\mathrm{ed}}$ : 


$$
C=\frac{s_{\mathrm{ob}}\left[2 \pi n_{\mathrm{ed}}-\sin \left(2 \pi n_{\mathrm{ed}}\right)\right]+\sqrt{\Delta}}{4 \pi n_{\mathrm{ed}}},
$$

where $\Delta=2 \pi n_{\mathrm{ed}} s_{\mathrm{ob}}^{2} \sin \left(2 \pi n_{\mathrm{ed}}\right) \sin ^{2}\left(\pi n_{\mathrm{ed}}\right)+2 \pi^{2} n_{\mathrm{ed}}^{2}\left(8-s_{\mathrm{ob}}^{2}\right)-s_{\mathrm{ob}}^{2}\left(1-\pi n_{\mathrm{ed}}\right) \sin \left(2 \pi n_{\mathrm{ed}}\right)$.

The equation for the bronchus cross section with obstructive constriction (6) has the form

$$
\begin{aligned}
& X_{n}^{B}(\varphi)=R_{n} \cos (\varphi) \cdot f(\varphi), \\
& Y_{n}^{B}(\varphi)=R_{n} \sin (\varphi) \cdot f(\varphi),
\end{aligned}
$$

where $\varphi \in[0,2 \pi]$ and $R_{n}$ is the radius of the bronchus (1) of the $n$-th generation.

Figure 10 shows the shapes of the starry inner cross section of the bronchi (solid curves) and the circular cross section of the bronchi (dashed curves). The circular and starry bronchi have an identical cross-sectional area in Figure 10,a, whereas the cross-sectional area of the starry bronchus in Figure 10,b is smaller than that of the circular bronchus.

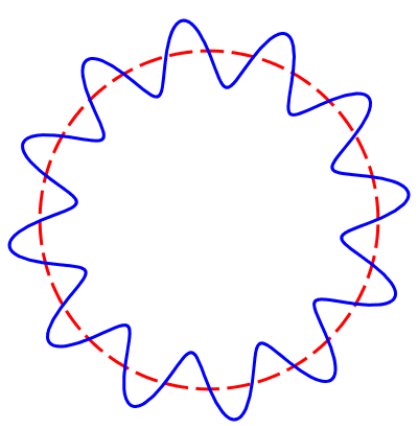

a)

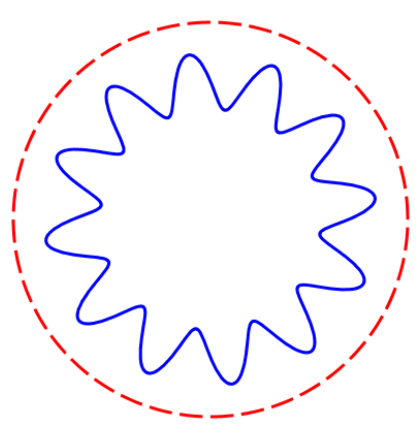

b)

Fig. 10. Shape of the inner structure of the bronchi calculated by formulas (6)-(8).

\section{ANALYTICAL DESIGN OF THE BRONCHIAL TREE}

The main problem in constructing the bifurcation of the bronchial tree is to ensure a correct description of the carinal rounding of the bifurcating bronchi. The quality of carinal rounding construction affects the quality of grid generation and, finally, the solution accuracy. Nonalgorithmic construction of the carinal rounding (e.g., simple smoothing of sharp ribs) requires many manual operations and attention in design of a branching bronchial tree.

An individual bifurcation was analytically designed for constructing the full human bronchial tree. Then, changing the bifurcation number, one can obtain the entire set of the bronchi. The bronchus position in the bronchial tree is also defined analytically with the use of coordinate transformations in accordance with the bronchus position in the tree.

\section{Construction of an individual bifurcation}

Design of an individual bifurcation is based on the idea [8] of using torus configurations. An individual $n$-th bifurcation of the bronchial tree is constructed from parts of two torus configurations and three-dimensional two-parameter surfaces smoothly connecting these configurations. The value of the bronchus deflection angle $\chi_{n}$ is not regulated in the bronchial tree model [1] (formulas (1), (2)); therefore, for certainty, we use a constant angle $\chi_{n}=35^{\circ}$, as in [8].

The equation for a torus with the distance from the circumference center to the rotation axis $R$ and with the circumference radius $r$ is defined parametrically by the formula 


$$
\begin{aligned}
& X(\varphi, \chi, R, r)=[R+r \cdot f(\varphi) \cos \varphi] \cos \chi, \\
& Y(\varphi, r)=r \cdot f(\varphi) \sin \varphi, \\
& Z(\varphi, \chi, R, r)=[R+r \cdot f(\varphi) \cos \varphi] \sin \chi,
\end{aligned}
$$

where the angles are $\varphi \in[0,2 \pi]$ and $\chi \in[-\pi, \pi]$, and $f(\varphi)$ is the function of the bronchus cross section shape (6)

To construct torus (9) with a variable circumference radius $R$, we need the equation for the curve connecting two points $\left(R_{0}, 0\right)$ and $\left(R_{1}, \chi_{n}\right)$ with the second order of smoothness

$$
R_{n}^{M}\left(\chi ; R_{0}, R_{1}\right)=\frac{R_{0}-R_{1}}{2} \sin \left(\pi\left(\frac{\chi}{\chi_{n}}+\frac{1}{2}\right)\right)+\frac{R_{0}+R_{1}}{2} .
$$

Figure 11 shows the general view (a) and the projection (b) of the right part of the bifurcation $(x>0)$ onto the plane $y=0$. The upper torus $\left(0, x_{a}, f_{a}, f_{c}\right)$ has the input radius $\left(0, x_{a}\right)=R_{n}$ and the output radius $\left(f_{b}, f_{c}\right)=\left(f_{c}, f_{a}\right)=R_{n+1}$, and is bounded by the angle $\chi_{n}$. The lower torus $\left(z_{a}, f_{c}, f_{b}, z_{c}\right)$ has the input and output radii $\left(z_{a}, z_{b}\right)=\left(f_{c}, f_{b}\right)=R_{n+1}$. The inner radius of the lower torus is $\left(z_{d}, z_{b}\right)=\left(z_{d}, f_{b}\right)=R_{n+1} / 2$. The distance is $\left(0, x_{b}\right)=G_{n}$. The parameter $G_{n}$ is defined by the relation

$$
G_{n}=\left(2.5 R_{n+1}-R_{n}\right) \frac{\cos \chi_{n}}{1-\cos \chi_{n}}
$$

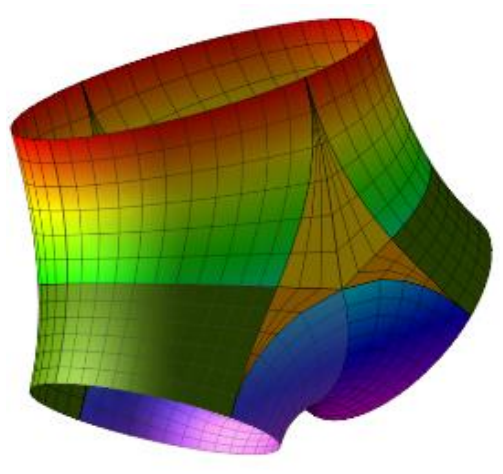

a)

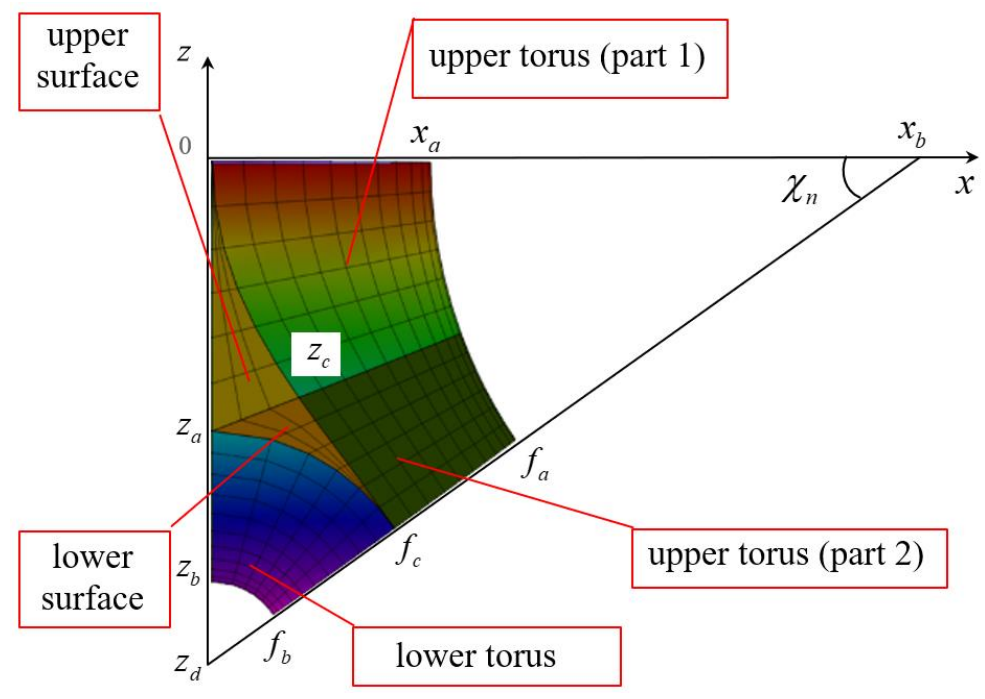

b)

Fig. 11. Construction of the $n$-th bifurcation of the bronchial tree (the picture illustrates the construction of a circular bifurcation, and the output bronchi are not shown). General view of the three-dimensional bifurcation (a). Projection in the plane $y=0$ (b). Here $\left(0, x_{a}, f_{a}, f_{c}\right)$ is the upper torus, $\left(z_{a}, f_{c}, f_{b}, z_{c}\right)$ is the lower torus, $\left(0, z_{e}, z_{a}\right)$ is the upper surface, and $\left(z_{a}, z_{e}, f\right)$ is the lower surface.

The upper torus $\left(0, x_{a}, f_{a}, f_{c}\right)$ is described by the relations 


$$
\begin{aligned}
& X_{n}^{U}(\varphi, \chi)= \pm\left[G_{n}+X\left(\varphi, \chi-\pi, R_{n}^{M}\left(\chi ; G_{n}, G_{n}-\left(R_{n}-R_{n+1}\right)\right), R_{n}^{M}\left(\chi ; R_{n}, R_{n+1}\right)\right)\right], \\
& Y_{n}^{U}(\varphi, \chi)=Y\left(\varphi, R_{n}^{M}\left(\chi ; R_{n}, R_{n+1}\right)\right), \\
& Z_{n}^{U}(\varphi, \chi)=Z\left(\varphi, \chi-\pi, R_{n}^{M}\left(\chi ; G_{n}, G_{n}-\left(R_{n}-R_{n+1}\right)\right), R_{n}^{M}\left(\chi ; R_{n}, R_{n+1}\right)\right),
\end{aligned}
$$

where $\varphi \in[\pi / 2,3 \pi / 2], \chi \in\left[0, \chi_{n}\right]$.

The lower torus $\left(z_{a}, f_{c}, f_{b}, z_{c}\right)$ is described by the relations

$$
\begin{aligned}
& X_{n}^{D}(\varphi, \zeta)= \pm X\left(\varphi, \zeta, 1.5 R_{n+1}, R_{n+1}\right), \\
& Y_{n}^{D}(\varphi, \zeta)=Y\left(\varphi, R_{n}^{M}\left(A_{n}(\zeta) ; R_{n}, R_{n+1}\right)\right), \\
& Z_{n}^{D}(\varphi, \zeta)=-G_{n} \tan \chi_{n}+Z\left(\varphi, \zeta, 1.5 R_{n+1}, R_{n+1}\right),
\end{aligned}
$$

where $\varphi \in[\pi / 2,3 \pi / 2], \zeta \in\left[\chi_{n}, \pi / 2\right]$.

The upper two-parameter surface $\left(0, z_{e}, z_{a}\right)$ is defined by the equations

$$
\begin{aligned}
& x_{n}^{U}(s)= \pm s, \\
& y_{n}^{U}(\chi)=Y_{n}^{U}( \pm \pi / 2, \chi), \\
& z_{n}^{U}(s, \chi)=\left(s-G_{n}\right) \tan \chi,
\end{aligned}
$$

where $s \in\left[0, X_{n}^{U}(\pi / 2, \chi)\right], \chi \in\left[0, \bar{\chi}_{n}\right]$. Here the angle $\bar{\chi}_{n}$ bounding the second parameter is defined as

$$
\bar{\chi}_{n}=-\arctan \left(\frac{1.5 R_{n+1}-G_{n} \tan \chi_{n}}{G_{n}}\right) .
$$

The lower two-parameter surface $\left(0, z_{e}, z_{a}\right)$ is defined by the equations

$$
\begin{aligned}
& x_{n}^{D}(s)= \pm s, \\
& y_{n}^{D}(\zeta)=Y_{n}^{U}\left( \pm \pi / 2, A_{n}(\zeta)\right), \\
& z_{n}^{D}(s, \zeta)=\left(s-G_{n}\right) \tan A_{n}(\zeta),
\end{aligned}
$$

where $s \in\left[X_{n}^{D}(\pi / 2, \zeta), X_{n}^{U}\left(\pi / 2, A_{n}(\zeta)\right)\right], \zeta \in\left[\chi_{n}, \pi / 2\right]$.

The function $A_{n}(\zeta)$ determines the relationship between the angles $\zeta$ and $\chi$ :

$$
A_{n}(\zeta)=\chi_{n}-\arctan \left(\frac{1.5 R_{n+1} \sin \left(\zeta-\chi_{n}\right)}{\frac{G_{n}}{\cos \chi_{n}}-1.5 R_{n+1} \cos \left(\zeta-\chi_{n}\right)}\right) .
$$

The coordinates of the point $f_{c}$ (center of the output bronchi) are

$$
\begin{aligned}
& x_{n}^{c}= \pm 1.5 R_{n+1} \cos \chi_{n}, \\
& y_{n}^{c}=0, \\
& z_{n}^{c}=-\left[G_{n}-\left(R_{n}-R_{n+1}\right)\right] \sin \chi_{n} .
\end{aligned}
$$


The surface $\left(f_{a}, f_{c}, f_{b}\right)$ is adjacent to the output cylindrical or starry bronchus of length $L_{n+1}$ (not shown in Fig. 11). The equations of the output bronchi are

$$
\begin{aligned}
& X_{n}^{B(\text { out })}(\varphi, s)=x_{n}^{c}+R_{n+1} f(\varphi) \cos (\varphi) \cos \chi_{n} \pm s \cdot \sin \chi_{n}, \\
& Y_{n}^{B(\text { out })}(\varphi, s)=R_{n+1} f(\varphi) \sin (\varphi), \\
& Z_{n}^{B(\text { out })}(\varphi, s)=z_{n}^{c} \pm R_{n+1} f(\varphi) \cos (\varphi) \sin \chi_{n}-s \cdot \cos \chi_{n},
\end{aligned}
$$

where the plus and minus signs refer to the right and left output bronchi, respectively, the parameter is $s \in\left[0, L_{n+1}\right]$, and the angle is $\varphi \in[0,2 \pi]$.

Equations (9)-(19) provide an analytical description of the construction of the $n$-th bifurcation of the bronchial tree with the output bronchi. Each bifurcation consists of the following 3D surfaces: 1) two upper toroidal surfaces (right and left surfaces indicated by the plus and minus signs, respectively) (formulas (12)); 2) two lower toroidal surfaces (right and left surfaces indicated by the plus and minus signs, respectively (formulas (13)); two upper twoparameter surfaces (right and left surfaces indicated by the plus and minus signs, respectively) (formulas (14)); two lower two-parameter surfaces (right and left surfaces indicated by the plus and minus signs, respectively (formulas (16)); output cylindrical or starry bronchi (right and left bronchi indicated by the plus and minus signs, respectively) (formulas (19)).

\section{Bronchial tree construction}

The next bifurcation is constructed in a similar manner. The design of the following bifurcations of the bronchial tree begins from the transformation of the coordinate system with the origin in the middle of the right or left output bronchus of the previous bifurcation. The $Z$ axis is directed along the axis of the left or right output bronchus of the previous bifurcation. This transformation of coordinates allows one to transfer the bifurcation to a needed point of the tree without changing the formulas for bronchus design (the only changes is in the bifurcation number $n$ ).

We pass to a new coordinate system from the current bifurcation when the origin of the coordinate axes is shifted to the point $\left(x_{n+1}^{\text {new }}, y_{n+1}^{\text {new }}, z_{n+1}^{\text {new }}\right)$ :

$$
\begin{aligned}
& x_{n+1}^{\text {new }}= \pm\left(1.5 R_{n+1} \cos \chi_{n}+L_{n+1} \sin \chi_{n}\right), \\
& y_{n+1}^{\text {new }}=0, \\
& z_{n+1}^{\text {new }}=-\left[G_{n}-\left(R_{n}-R_{n+1}\right)\right] \sin \chi_{n}-L_{n+1} \cos \chi_{n}
\end{aligned}
$$

and rotated around the $Y$ axis by the angle $\pm \chi_{n}$.

Beginning from the second bifurcation $(n=2)$, even bifurcations are rotated around the $Z$ axis (here we mean the local coordinate system in which the bifurcation is constructed) by the angle $\pi / 2$. As a result, the matrix of the coordinate transformations for the next bifurcation with the number $n+1$ has the form:

- for odd numbers $n$

$$
S_{n+1}=\left[\begin{array}{cccc}
\cos \left(\chi_{n}\right) & 0 & \mp \sin \left(\chi_{n}\right) & 0 \\
0 & 1 & 0 & 0 \\
\pm \sin \left(\chi_{n}\right) & 0 & \cos \left(\chi_{n}\right) & 0 \\
\pm x_{n+1}^{\text {new }} & y_{n+1}^{\text {new }} & z_{n+1}^{\text {new }} & 1
\end{array}\right]
$$

- for even numbers $n \geq 2$ 


$$
S_{n+1}=\left[\begin{array}{cccc}
0 & \cos \left(\chi_{n}\right) & \mp \sin \left(\chi_{n}\right) & 0 \\
-1 & 0 & 0 & 0 \\
0 & \pm \sin \left(\chi_{n}\right) & \cos \left(\chi_{n}\right) & 0 \\
-y_{n+1}^{\text {new }} & \pm x_{n+1}^{\text {new }} & z_{n+1}^{\text {new }} & 1
\end{array}\right] .
$$

Figure 12, a shows the bronchial tree constructed up to the generation $n=5$ with the starry cross-sectional shape of the bronchi. Bifurcations of the same generation are marked by an identical color. It is impossible to show the full bronchial tree up to the 23-rd generation because there are too many bifurcations of versatile scales. As an example, Figure 12,b shows one branch of the bronchial tree (only bifurcations adjacent to the right output bronchi) up to the 23-rd generation inclusive.

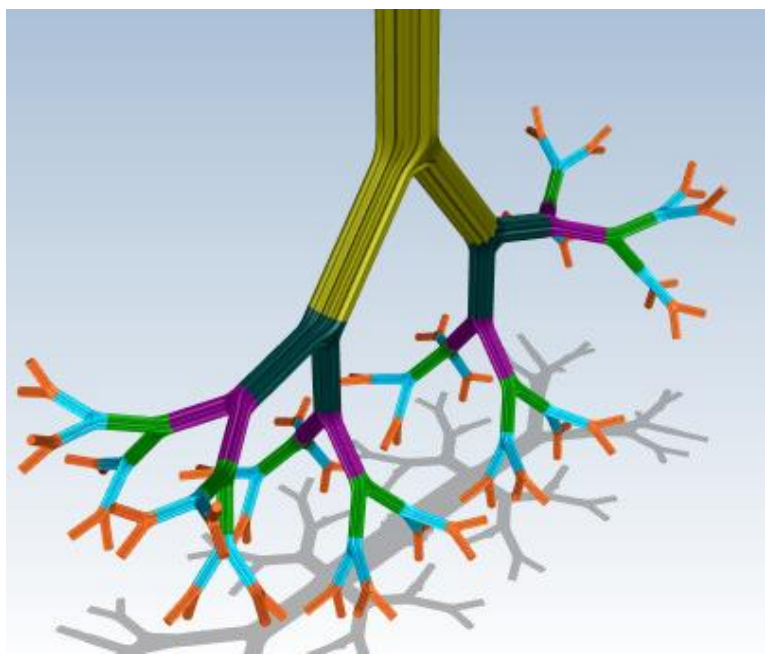

a)

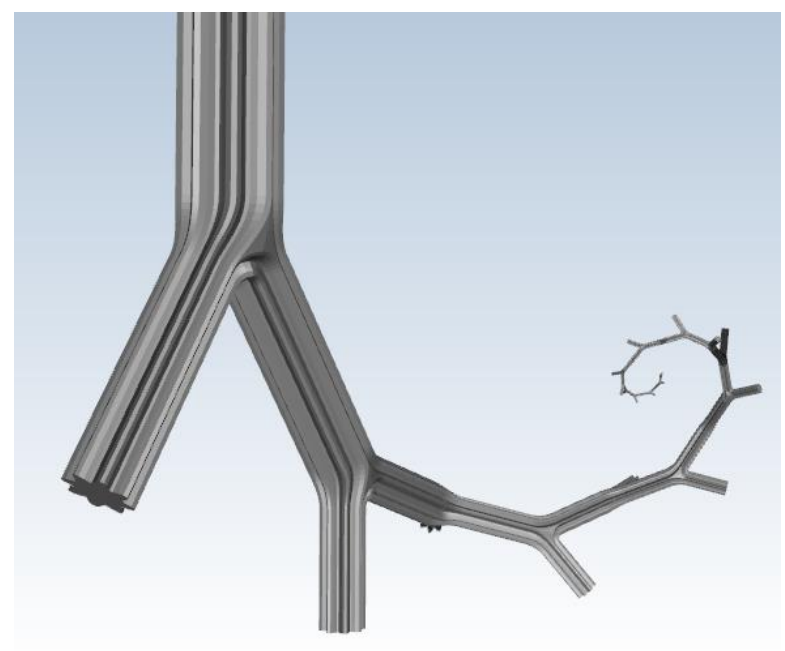

b)

Fig. 12. Patterns of the human bronchial tree with starry bronchi designed on the basis of the proposed model (9)-(22). (a) Bronchial tree up to the generation $n=5$ (branches of one generation are marked by the same color). (b) One branch of the bronchial tree with generations from 0 to 23.

\section{CONCLUSIONS}

The paper describes the analytical design of the full human bronchial tree for healthy persons and patients with obstructive pulmonary diseases. The existing methods of bronchial tree design have an empirical character and are restricted by the capabilities of complex geometry construction by a particular commercial CDF software system. The available models of the bronchial tree do not describe the starry internal structure of bronchioles; thus, they fail to describe lungs with pathological effects.

An analytical model is developed for constructing lungs with an arbitrary number of generations (up to alveoli). The analytical model of the bifurcation contains closed formulas for constructing the full human bronchial tree from the 0-th to 23-rd bifurcation inclusive. Analytical formulas allow easy modeling of lung system pathologies caused by starry constriction of the bronchi. The geometric characteristics of the human bronchial tree in pathological cases are modeled by a starry internal structure of the bronchus, and the degree of pathology is defined by two parameters: degree of bronchus constriction and degree of distortion of the cylindrical shape of the bronchus. All surfaces of the bronchial tree are matched with the second order of smoothness (have no acute angles or sharp ribs). Moreover, analytical formulas allow one to avoid tedious manual operations of constructing each individual bifurcation in 3D simulation codes, their matching and smoothing (the procedure of manual design of the pathological bifurcation is especially complicated). The proposed analytical 
formulas of bronchial tree construction allow numerical simulations to be performed for bronchial trees with an arbitrary number of generation (provided that appropriate computational resources are available) for normal and pathological cases.

The study was supported by the Russian Foundation for Basic Research and the Subject of the Russian Federation (Novosibirsk Region) within the framework of the research project No. 19-41-540003 r_a.

\section{REFERENCES}

1. Weibel E.R. Morphometry of the Human Lung. Berlin: Springer Verlag, 1963.

2. Zhao Y., Lieber B.B. Steady inspiratory flow in a model symmetric bifurcation. Journal of Biomechanical Engineering. 1994. V. 116. P. 488-496. doi: 10.1115/1.2895800.

3. Zhao Y., Brunskill C.T., Lieber B.B. Inspiratory and expiratory steady flow analysis in a model symmetrically bifurcating airway. Journal of Biomechanical Engineering. 1997. V. 119. P. 52-58. doi: 10.1115/1.2796064.

4. Hegedüs C.J., Balásházy I., Farkas Á. Detailed mathematical description of the geometry of airway bifurcations. Respiratory Physiology \& Neurobiology. 2004. V. 141. No. 1. P. 99-114. doi: 10.1016/j.resp.2004.03.004.

5. Heistracher T., Hofmann W. Physiologically realistic models of bronchial airway bifurcations. J. Aerosol Sci. 1995. V. 26. No. 3. P. 497-509. doi: $\underline{10.1016 / 0021-}$ 8502(94)00113-D.

6. Ertbruggen C., Hirsch C., Paiva M. Anatomically based three-dimensional model of airways to simulate flow and particle transport using computational fluid dynamics. J. Appl. Physiol. 2005. V. 98. P. 970-980. doi: 10.1152/japplphysiol.00795.2004.

7. Tena A.F., Casan P., Fernández J., Ferrera C., A. Marcos A. Characterization of particle deposition in a lung model using an individual path. EPJ Web of Conferences. 2013. V. 45. Article No. 01079. doi: 10.1051/epjconf/20134501079.

8. Tena A.F., Fernández J., Álvarez E., Casan P., Walters D.K. Design of a numerical model of lung by means of a special boundary condition in the truncated branches. International Journal for Numerical Methods in Biomedical Engineering. 2017. V. 33. No. 6. Article No. e2830. doi: 10.1002/cnm.2830.

9. Tena A.F., Francos J.F., Álvarez E., Casan P.A. A three dimensional in SILICO model for the simulation of inspiratory and expiratory airflow in humans. Engineering Applications of Computational Fluid Mechanics. 2015. V. 9. No. 1. P. 187-198. doi: 10.1080/19942060.2015.1004819.

10. Gemci T., Ponyavin V., Chen Y., Chen H., Collins R. CFD Simulation of Airflow in a 17-Generation Digital Reference Model of the Human Bronchial Tree. Series on Biomechanics. 2007. V. 23. No. 1. P. 5-18.

11. Gemci T., Ponyavin V., Chen Y., Chen H., Collins R. Computational model of airflow in upper 17 generations of human respiratory tract. Journal of Biomechanics. 2008. V. 41. P. 2047-2054. doi: 10.1016/j.jbiomech.2007.12.019.

12. Trusov P.V., Zaitseva N.V., Tsinker M.Yu. Modeling of the human breathing process: conceptual and mathematical formulations. Mathematical Biology and Bioinformatics. 2016. V. 11. No. 1. C. 64-80. doi: 10.17537/2016.11.64.

13. Trusov P.V., Zaitseva N.V., Tsinker M.Yu., Babushkina A.V. Modelling dusty air flow in the human respiratory tract. Russian Journal of Biomechanics. 2018. V. 22. No. 3. P. 262-274.

14. Choi J. Multiscale numerical analysis of airflow in CT-based subject specific breathing human lungs: PhD Dissertation (Doctor of Philosophy). Iowa: University of Iowa, 2011. 259 p. doi: 10.17077/etd.n7qno7h9.

15. Ham A.W., Cormack D.H. Ham's Histology. Philadelphia: Lippencott, 1979. 
16. Mescher A.L. Junqueira's Basic Histology: Text and Atlas. New York: McGraw Hill Medical, 2013. 560 p.

17. Chernyaev A.L., Samsonova M.V. Different types of chronic obstructive pulmonary disease in term of pathologist's view. Russian Pulmonology. 2013. No. 3. P. 93-96 (in Russ.). doi: 10.18093/0869-0189-2013-0-3-93-96.

18. Solopov V.N. Astma. Kak vernut' zdorov'e (Asthma. How to be healthy again). 2002. 240 p. (in Russ.). URL: http://health.astma.ru/ (accessed 18.12.2019). 